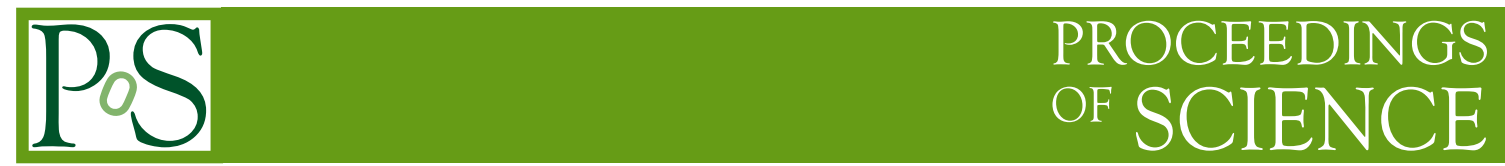

\title{
Comparative study of topological charge
}

\section{Yusuke Namekawa*}

Center for Computational Sciences, University of Tsukuba, Tsukuba, Ibaraki 305-8577, Japan

E-mail: namekawa@ccs.tsukuba.ac.jp

Comparative study of topological charge is performed. Topological charges are measured by a cloverleaf operator on smoothed gauge configurations. Various types of smoothing techniques are employed. Agreement of topological charges in fermionic and gluonic definitions is examined. High consistency is observed between topological charges obtained by improved smoothing methods and those by the index theorem with the overlap-Dirac operator.

The 32nd International Symposium on Lattice Field Theory,

23-28 June, 2014

Columbia University New York, $N Y$

${ }^{*}$ Speaker. 


\section{Introduction}

A topological charge is one of the most fundamental quantity in QCD. It characterizes the vacuum structure. Lattice QCD is a main tool in the study of the topological charge [1]. Lattice QCD allows us to perform a nonperturbative analysis in a systematic way.

The topological charge is often measured with a gluonic field strength operator on the lattice. Though it suffers from noisy ultraviolet fluctuations, a smoothing technique tames them so that a discernible signal of the topological charge can be obtained. Cooling or smearing have been used for smoothing gauge fields. Recently, a gradient flow is also employed. In contrast to the traditional cooling and smearing, the gradient flow has an advantage that it provides a continuous change of the gauge field. The gradient flow accomplishes a better control of smoothing.

Alternatively, the topological charge can be calculated with a fermionic definition. The topological charge is determined, for example, by the index theorem with the overlap-Dirac operator. A clear advantage of the fermionic definition is that the result is guaranteed to be an integer. A subtle point, on the other hand, is the integer values depend on the choice of the definition, due to a finite lattice spacing. In the case of the overlap-Dirac operator, a value of the topological charge occasionally changes according to a parameter in the formulation. Consistency check of the topological charges in the fermionic and gluonic definitions would be helpful as an estimator of the scaling violation.

In this work, topological charges are computed with gluonic operators on $N_{f}=2$ topology fixed gauge configurations. The measurements are performed using several smoothing techniques. Cooling with plaquette and improved local actions, APE and HYP smearing, as well as gradient flows are employed. The results are compared with each other, and with the values obtained using the overlap-Dirac operator. Similar attempts are reported in Refs. [2].

\section{Setup}

\subsection{Gauge configuration}

Measurement of the topological charge is performed on $N_{f}=2$ gauge configurations provided by JLQCD Collaboration [3]. The lattice size is $16^{3} \times 32$ at the lattice spacing of $a=0.118(2) \mathrm{fm}$. The gluon action is Iwasaki-type improved gauge action,

$$
S_{g}=\beta\left(c_{0}^{g} \sum_{x, \mu<v} P_{\mu v}(x)+c_{1}^{g} \sum_{x, \mu, \nu} R_{\mu v}(x)\right),
$$

where $\beta=6 / g_{0}^{2}, c_{0}^{g}=3.648, c_{1}^{g}=-0.331$. The quark action is an overlap-Dirac fermion action,

$$
\begin{aligned}
S_{q} & =\bar{q} D_{\mathrm{ov}}(m) q, \\
D_{\mathrm{ov}}(m) & =\left(m_{0}+\frac{m}{2}\right)+\left(m_{0}-\frac{m}{2}\right) \gamma_{5} \operatorname{sgn}\left(H_{\mathrm{W}}\left(-m_{0}\right)\right), H_{\mathrm{W}}\left(-m_{0}\right)=\gamma_{5} D_{\mathrm{W}}\left(-m_{0}\right),
\end{aligned}
$$

where $m$ is the bare quark mass. $D_{\mathrm{W}}\left(-m_{0}\right)$ is the Wilson operator with a negative mass, $-m_{0}=$ -1.6. Furthermore, unphysical Wilson fermion $\psi$ with a negative mass as well as twisted mass terms are added to fix the topological charge defined by the index theorem $Q_{\text {index }}$,

$$
\delta S_{\mathrm{W}}=\bar{\psi} D_{\mathrm{W}}\left(-m_{0}\right) \psi+\phi^{\dagger}\left(D_{\mathrm{W}}\left(-m_{0}\right)+i \mu \gamma_{5} \tau_{3}\right) \phi
$$




\begin{tabular}{ccccc}
\hline$\beta$ & $m$ & $Q_{\text {index }}$ & \# conf & MD time \\
\hline 2.30 & 0.05 & -2 & 50 & 2500 \\
\hline
\end{tabular}

Table 1: Parameters of the gauge configurations. Molecular Dynamics time is the number of trajectories multiplied by the trajectory length.

where $\phi$ is a pseudofermion, and $\mu$ is the twisted mass parameter. $\mu=0.2$ is employed in the configuration generation. Each configuration is separated by 100 trajectories with its trajectory length 0.5. The main simulation parameters are summarized in Table 1.

\subsection{Gluonic topological charge operator}

The topological charge is measured using a gluonic field strength.

$$
\begin{aligned}
Q_{\text {improve }} & =c_{0} Q^{1 \times 1}+c_{1} Q^{1 \times 2}, \\
Q^{1 \times 1,2} & =\frac{1}{32 \pi^{2}} \sum_{\mu, v, \rho, \sigma} \varepsilon_{\mu v \rho \sigma} \operatorname{Tr} F_{\mu \nu}^{1 \times 1,2} F_{\rho \sigma}^{1 \times 1,2}, \\
F_{\mu \nu}^{1 \times 1,2} & =-\frac{i}{4}\left[C_{\mu \nu}^{1 \times 1,2}\right]^{\mathrm{AH}}, C_{\mu \nu}^{\mathrm{AH}}=\frac{1}{2 i}\left(C_{\mu \nu}-C_{\mu \nu}^{\dagger}\right),
\end{aligned}
$$

where $C_{\mu \nu}^{1 \times 1}$ is the cloverleaf constructed with $1 \times 1$ plaquettes, and $C_{\mu \nu}^{1 \times 2}$ with $1 \times 2$ rectangular loops. The improvement coefficients $c_{0}$ and $c_{1}$ can be tuned to reduce the scaling violation in the topological charge operator. Three types of $\left(c_{0}, c_{1}\right)$ are investigated.

$$
\begin{aligned}
\left(c_{0}, c_{1}\right) & =(1,0) \quad \text { Naive-type, } \\
& =(5 / 3,-1 / 12) \quad \text { Symanzik-type }, \\
& =(3.648,-0.331) \quad \text { Iwasaki-type. }
\end{aligned}
$$

Figure 1 displays $c_{1}$ dependence of the topological charge on a single configuration smoothed by Wilson flow. The topological charge with Symanzik-type coefficients has the smallest deviation from an integer. Since the deviation is originated from the finite lattice spacing, it implies an efficient reduction of the scaling violation by Symanzik-type operator. Calculations on other configurations show a similar tendency. Based on this result, Symanzik-type coefficients are employed in this work.

\subsection{Smoothing}

Three kinds of smoothing techniques are evaluated: cooling, smearing, and gradient flow. Smoothing is required to suppress noisy ultraviolet fluctuations, while keeping a topological structure. Although any smoothing is expected to give a consistent result in the continuum limit, it is valuable to find a method that has the least lattice artifact.

Cooling eliminates ultraviolet noises by replacing each link variable such that the local action is minimized [4]. For the local action, not only a naive plaquette action, but also Symanzik and Iwasaki actions are employed with the coefficients of Eq. (2.8)-(2.10). 


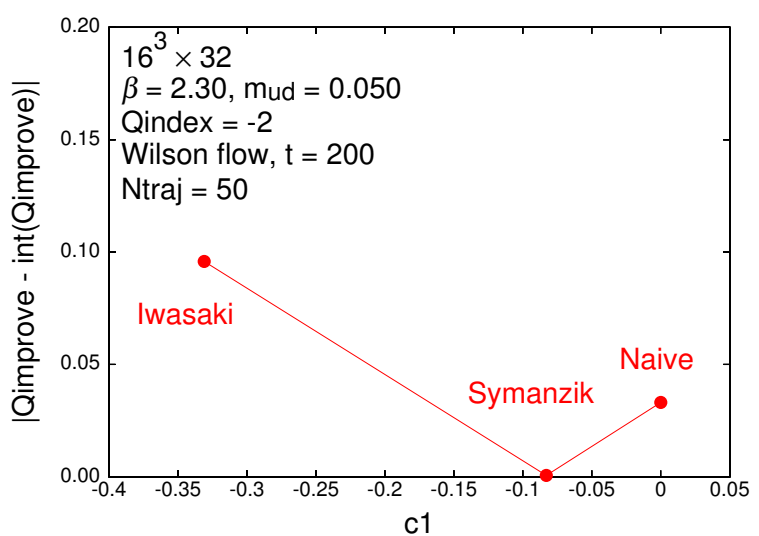

Figure 1: Improvement coefficient $c_{1}$ dependence of improved topological charge $Q_{\text {improve }}$ on a single gauge configuration. Difference of $Q_{\text {improve }}$ from an integer is plotted.

Another way of smoothing is smearing. APE smearing [5] is defined by

$$
\begin{aligned}
U_{\mu}^{\text {new }}(x) & =\operatorname{Proj}_{\mathrm{SU}(3)}\left[(1-\alpha) U_{\mu}(x)+\frac{\alpha}{6} \Sigma_{\mu}(x)\right], \alpha=0.6 . \\
\Sigma_{\mu}(x) & =\sum_{ \pm v \neq \mu} U_{v}(x) U_{\mu}(x+v) U_{v}^{\dagger}(x+\mu)
\end{aligned}
$$

In addition, HYP smearing [6] is also examined.

$$
\begin{aligned}
U_{\mu}^{\text {new }}(x) & =\operatorname{Proj}_{\mathrm{SU}(3)}\left[\left(1-\alpha_{1}\right) U_{\mu}(x)+\frac{\alpha_{1}}{6} \sum_{ \pm v \neq \mu} U_{v ; \mu}^{(2)}(x) U_{\mu ; v}^{(2)}(x+v) U_{v, \mu}^{(2), \dagger}(x+\mu)\right], \\
U_{\mu ; v}^{(2)}(x) & =\operatorname{Proj}_{\mathrm{SU}(3)}\left[\left(1-\alpha_{2}\right) U_{\mu}(x)+\frac{\alpha_{2}}{4} \sum_{ \pm \rho \neq \mu, v} U_{\rho ; \mu v}^{(3)}(x) U_{\mu ; v \rho}^{(3)}(x+\rho) U_{\rho, \mu v}^{(3), \dagger}(x+\mu)\right] \\
U_{\mu ; v \rho}^{(3)}(x) & =\operatorname{Proj}_{\mathrm{SU}(3)}\left[\left(1-\alpha_{3}\right) U_{\mu}(x)+\frac{\alpha_{3}}{2} \sum_{ \pm \sigma \neq \mu, v, \rho} U_{\sigma}(x) U_{\mu}(x+\sigma) U_{\sigma}^{\dagger}(x+\mu)\right], \\
\alpha_{1} & =0.75, \alpha_{2}=0.6, \alpha_{3}=0.3 .
\end{aligned}
$$

Smeared gauge fields are projected back to $S U(3)$ by Maximum $S U(3)$ projection.

$$
\operatorname{Proj}_{\mathrm{SU}(3)}^{\operatorname{maxSU}(3)}\left(U_{\mu}(x)\right)=\max _{U_{\mu}^{\text {new }}(x) \in S U(3)} \operatorname{Re} \operatorname{Tr}\left(U_{\mu}^{\text {new }}(x) U_{\mu}^{\dagger}(x)\right) .
$$

An alternative smoothing is given by the gradient flow [7]. The evolution of the gauge field is determined by

$$
\begin{aligned}
\partial_{t} V_{\mu}(x, t) & =-V_{\mu}(x, t) \frac{\partial S}{\partial V_{\mu}(x)}, \\
V_{\mu}(x, t=0) & =U_{\mu}(x),
\end{aligned}
$$

where $t$ is the flow time, and $S$ is an action without its coupling constant. Similar to the cooling case, plaquette, Symanzik, and Iwasaki actions are employed. The flow equation is solved by the fourth order Runge-Kutta in the commutator-free method [8]. The Runge-Kutta step size $d t$ is chosen to be 0.02 . The systematic error associated with discretization of the flow time is definitely below the statistical error. 

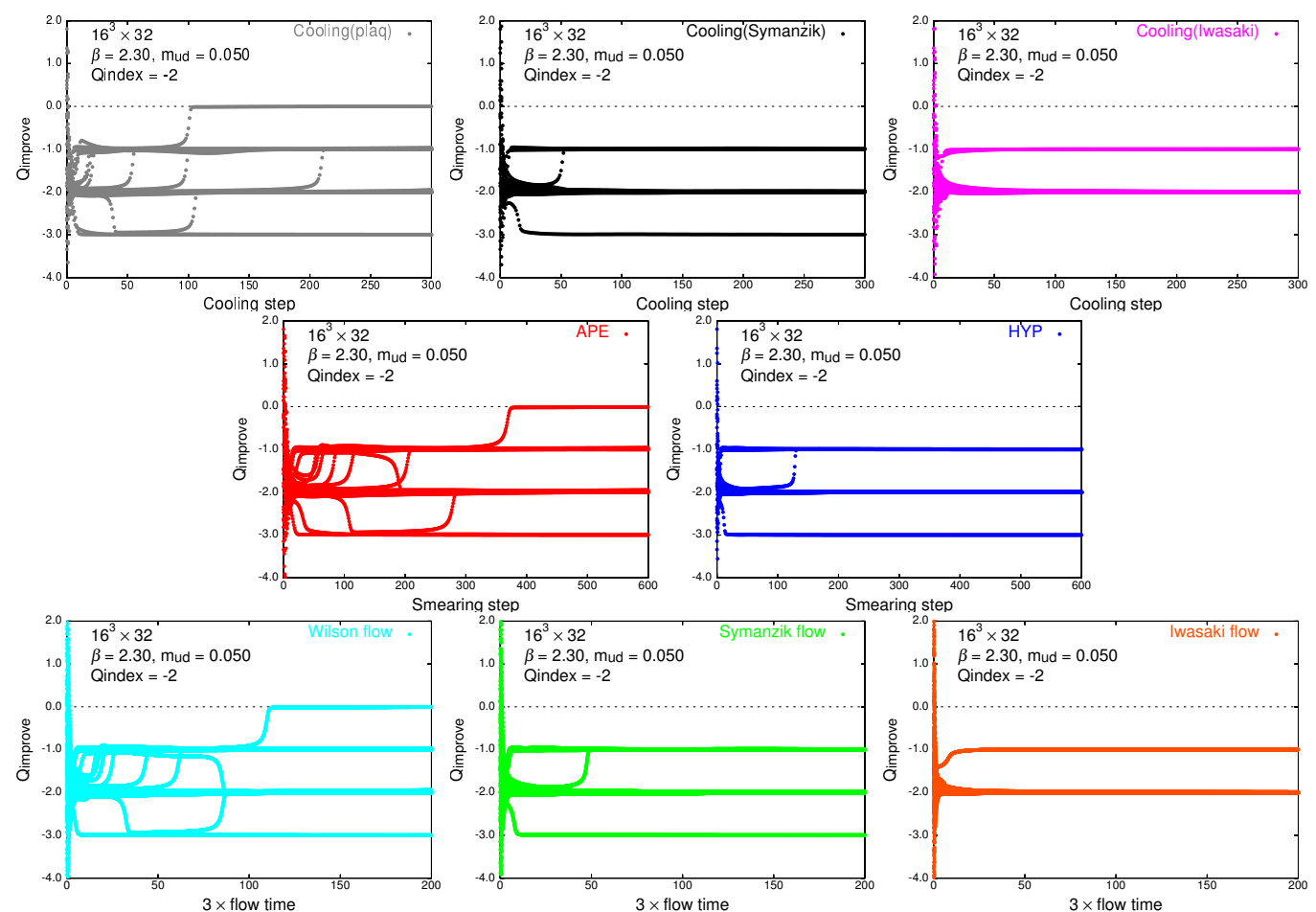

Figure 2: Smoothing step dependence of $Q_{\text {improve }}$ using cooling (top panels), smearing (middle panels), and gradient flow (bottom panels).

\subsection{Results}

Figure 2 illustrates cooling and smearing step dependence of the improved topological charge. The flow time dependence is also plotted. The flow time is multiplied by a factor of three, which is expected from a perturbative analysis [9]. In every case, the topological charge has an integer value with a sufficiently large number of steps. A small number of smoothing steps leads to a fake plateau i.e. a semi-stable value of the topological charge. The number of smoothing steps is determined to satisfy the admissibility condition, $\max \left[\operatorname{Re} \operatorname{Tr}\left(1-U_{\text {plaq }}\right)\right]<0.067$ [10, 11]. In Fig. 3, Wilson flow time dependence of the plaquette is shown. No jump of the topological charge seem to be triggered, if the admissibility condition is fulfilled. It should be mentioned $\max \left[\operatorname{Re} \operatorname{Tr}\left(1-U_{\text {plaq }}\right)\right]$ does not always decrease as the flow time grows, though the value summed over the spacetime falls off monotonically.

Figure 4 presents histograms of the improved topological charges. Since the topological charge determined by the index $Q_{\text {index }}$ is fixed in the configuration generations, the histogram is expected to have a sharp peak around $Q_{\text {index }}$, supposing the scaling violation is small. The histograms obtained by cooling with improved local actions show the expected behavior. Almost all of the topological charges agree with $Q_{\text {index }}$. On the other hand, cooling using the plaquette action has a broad histogram. It implies a relatively large lattice artifact in the unimproved cooling method. Analogous trends are observed in other smoothing procedures. HYP smearing has a narrow histogram, while APE smearing does not. Symanzik and Iwasaki flows form a sharp peak in the histogram. On the contrary, Wilson flow brings a wide peak. Improved smoothing methods leads to higher consis- 

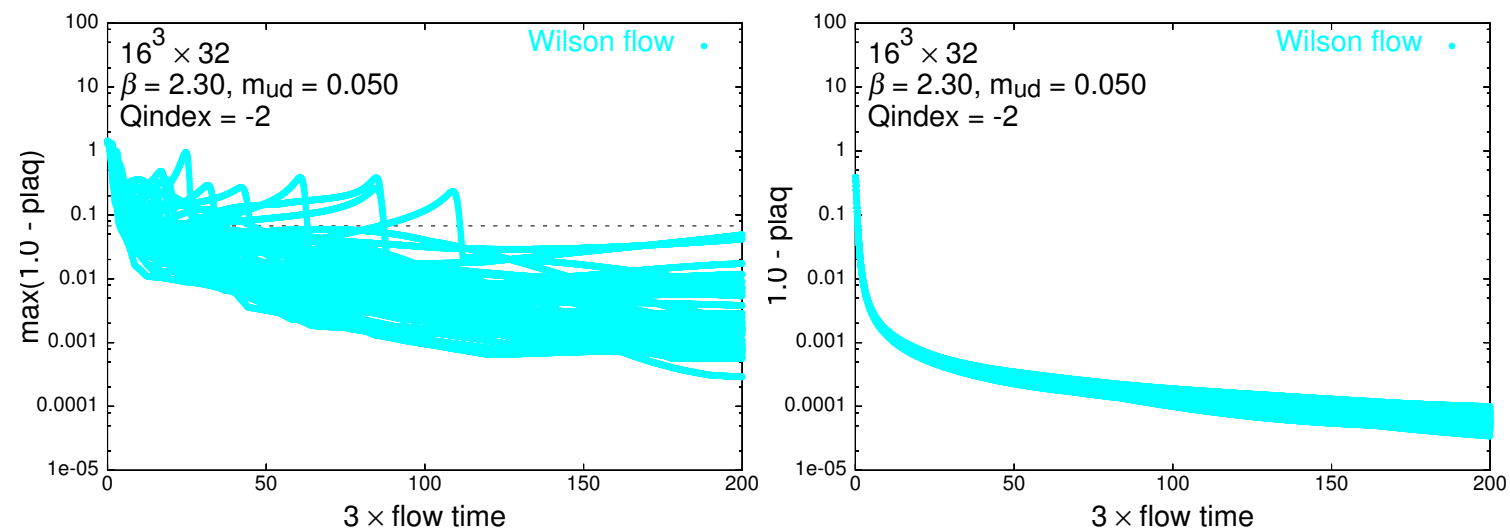

Figure 3: Wilson flow time dependence of maximum of (1 - plaquette) (left panel), and the value summed over the spacetime volume (right panel).

tency with $Q_{\text {index }}$, indicating the scaling violation in the topological charge is suppressed well by the improvement.

\subsection{Conclusion}

Systematic comparison of topological charges is presented. Topological charges are measured on $N_{f}=2$ topology fixed configurations. Several smoothing techniques are evaluated using a gluonic topological charge operator of Symanzik-type coefficients, which give a topological charge with the smallest deviation from an integer.

Cooling with improved actions, HYP smearing, and improved gradient flows are found to be advantageous. More than $90 \%$ of the topological charges are consistent with those obtained by the index theorem. It indicates their lattice artifacts are reduced efficiently. On the other hand, cooling with plaquette action, APE smearing, and Wilson flow lead to partial matches. The agreement is limited to $70-80 \%$. Scaling violations seem to be comparatively large in these smoothing methods.

Scaling properties as well as finite size effects of the topological charge have not been investigated. It is important to estimate them, but is beyond the scope of this work due to limitation of the gauge configurations. The gauge configurations have been generated at a single lattice spacing and spatial volume. These evaluations are left for the future work.

\section{Acknowledgments}

I would like to thank members of MEXT SPIRE Field 5 Project 1 and Bridge++ development team, as well as H.Fukaya and K-I.Nagai for valuable discussions. I am grateful to JLQCD Collaboration for providing their gauge configurations [3]. This work is in part based on Bridge++ code $[12,13,14]$. This work is supported by JLDG constructed over SINET3 of NII, MEXT SPIRE and JICFuS, and Grants-in-Aid for Scientific Research Grant Number 24540250.

\section{References}

[1] For a recent review, see M. Mueller-Preussker, PoS (LATTICE2014), 003 (2014). 


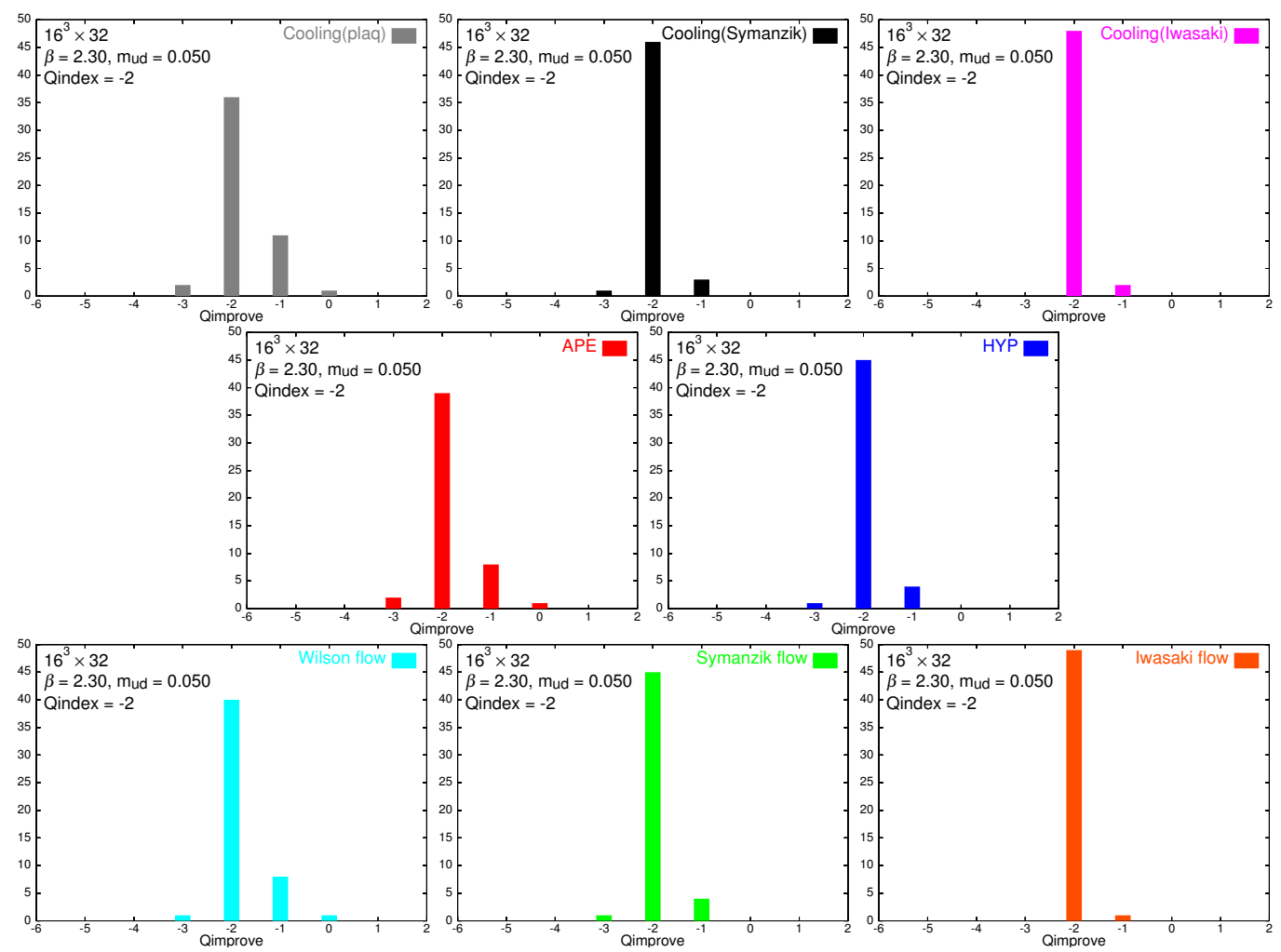

Figure 4: Histograms of $Q_{\text {improve }}$ with cooling (top panels), smearing (middle panels), and gradient flow (bottom panels).

[2] K. Cichy et al., PoS (LATTICE2014), 017 (2014); P. Giudice et al., PoS (LATTICE2014), 273 (2014).

[3] JLQCD Collaboration: S. Aoki et al., Phys. Rev. D78, 014508 (2008).

[4] B. Berg, Phys. Lett. 104B, 475 (1981); M. Teper, Phys. Lett. 162B, 357 (1985); J. Hoek et al., Rutherford preprint RAL-85-042 (1985); E. M. Ilgenfritz et al., Nucl. Phys. B268, 693 (1986).

[5] APE Collaboration: M. Albanese et al., Phys. Lett. B192, 163 (1987).

[6] A. Hasenfratz and F. Knechtli, Phys. Rev. D64, 034504 (2001).

[7] M. Lüscher, Commun. Math. Phys. 293, 899 (2010).

[8] E. Celledoni et al., FGCS 19, 341 (2003).

[9] C. Bonati and M. D’Elia, Phys. Rev. D89, 105005 (2014).

[10] M. Lüscher, Commun. Math. Phys. 85, 39 (1982); ibid. JHEP 08, 071 (2010).

[11] A. Phillips and D. Stone, Commun. Math. Phys. 103, 599 (1986).

[12] http://bridge.kek.jp/Lattice-code/

[13] S. Ueda et al., PoS (LATTICE2013), 412 (2014); PoS (LATTICE2014), 036 (2014); ibid. J. Phys. Conf. Ser. 523, 012046 (2014).

[14] S. Motoki et al., Proc. Comp. Sci. 29, 1701 (2014). 